\title{
Planar, Time-Optimal, Rest-to-Rest Slewing Maneuvers of Flexible Spacecraft
}

\author{
G. Singh, P. T. Kabamba, N. H. McClamroch \\ University of Michigan, Ann Arbor, Michigain
}

\begin{abstract}
The control problem of time-optimal, rest-to-rest slewing of a flexible spacecraft through a large angle is considered. The flexible spacecraft is modeled as a linear, elastic, undamped, nongyroscopic system suitable for analysis of planar rotational maneuvers. Minimum-time open-loop planar maneuvers are studied. The control histories are found to be bang-bang with multiple switches in each control variable. The optimal control history is shown to have an important time symmetry property. The switching times, final time, and costates at midmaneuver satisfy a system of nonlinear algebraic equations that can be solved using a homotopy method. An upper bound on attitude error due to control spillover is obtained. This helps to determine, a priori, the number of vibrational modes that need to be actively suppressed at the final time such that a prespecified pointing accuracy is guaranteed after the maneuver has been completed. A time-optimal slewing example is discussed to demonstrate the applicability of the results.
\end{abstract}

\section{Introduction}

$\mathbf{A}$ TTITUDE controllers for spacecraft have been based on the assumption that the bodies being controlled are rigid. However, future spacecraft may be quite flexible compared with their current counterparts. Many proposed applications require maneuvering these vehicles between two states of rest. In some time-critical applications, these maneuvers must be performed as rapidly as possible between two widely spaced quiescent attitudes. It is the design of a control system for such an application, using nonlinear control elements such as on-off actuators, that is considered in this work.

Large angle, three-axis, rotational maneuvers of spacecraft are usually performed by means of a sequence of single-axis rotations. Such maneuvers may take a relatively long time and their duration can often be reduced considerably by performing simultaneous three-axis slews. These maneuvers are necessarily more complex due to the inherent nonlinearities, even for a rigid spacecraft. Nonlinear effects are further compounded, for the flexible spacecraft case, when even small elastic deformations are considered, caused by rotational stiffening. This problem is not only infinite dimensional but also highly nonlinear. The problem of planar or single-axis rotational maneuvers is linear for the rigid spacecraft case; but for the elastic spacecraft case, kinematic nonlinearities are significant at high rotational rates; resulting in a nonlinear infinite dimensional problem.

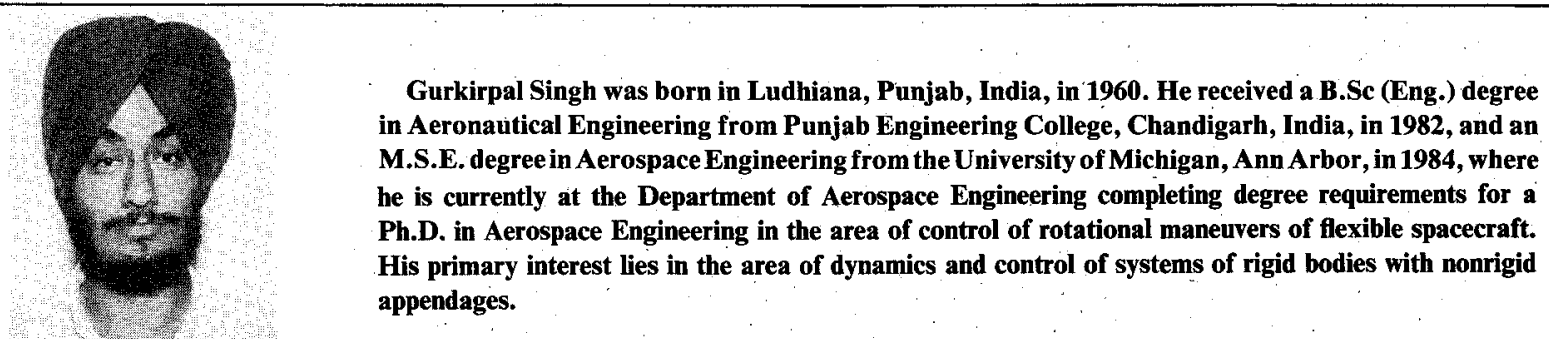

Pierre T. Kabamba was born in Lubumbashi (Zaire) in 1955. He received a Diplome d'Ingenieur Civil en Mathematique Appliquees from the University of Louvain, Belgium, in 1977, and a Ph.D. in Mechanical Engineering from Columbia University in 1981. He has held research and teaching positions at the University of Louvain (1977-1979 and 1981-1983) and Columbia University (1979-1981). Since 1983, he has been with the Aerospace Engineering Department at the University of Michigan, Ann Arbor, where he is an Assistant Professor. His interests include dynamics, reduced order control, model reduction, large space structures, guidance and navigation.

\footnotetext{
N. Harris McClamroch received degrees in mechanical engineering and in engineering mechanics from the University of Texas. Since 1967 he has been at the University of Michigan, Ann Arbor, where he is currently a Professor in the Department of Aerospace Engineering; he also holds a joint appointment with the Department of Electrical Engineering and Computer Science. His research interests are in the area of stability and control of nonlinear feedback systems, and include applications to control of mechanical systems. He is the author of numerous technical papers and one book. He is currently the Associate Editor for the IEEE Transactions on Automatic Control with responsibility for technical notes and correspondence.
}

Received June 1, 1987; revision received Aug. 13, 1987. Copyright (C) American Institute of Aeronautics and Astronautics, Inc., 1987. All rights reserved. 
Problems of rest-to-rest slewing maneuvers of rigid bodies have received considerable attention in the past. Euler's theorem on rotations has been widely used to obtain control histories for performing simultaneous three-axis slews. ${ }^{1-4,7}$ Kranton ${ }^{4}$ has studied near minimum-time maneuvers that are constrained so that the angular velocity vector remains fixed in the rigid body as well as in inertial space. It is unlikely that this occurs unless the desired change in orientation can be achieved by rotating the body about one of the principle axis. Various control schemes have used internal angular momentum management to bring about a desired change in orientation. $8,10,13,15,16$ Carrington and Junkins ${ }^{9}$ have solved the large angle maneuver problem using a polynomial feedback form by expanding the control as a polynomial function of system states with coefficients determined by minimizing a quadratic cost functional; the maneuver time was fixed in this problem. Nonlinear state and control transformations have also been employed to linearize the dynamical model. ${ }^{11-14,33}$ A reorientation problem was then formulated as a linear optimal control problem in the transformed space. Control torques were obtained by minimizing a quadratic cost functional. Quite recently ${ }^{18}$ variable structure control theory was used in studying rigid body slewing. Once again the control torques were obtained by minimizing a quadratic performance index. Dixon et al. ${ }^{5}$ used impulsive torques to perform minimum fuel reorientations of rigid spacecraft using a series of pulses and coasting arcs. Bergman et al. ${ }^{6}$ employed reaction control and used a linear programming algorithm for jet selection to perform rigid spacecraft rotational maneuvers. In another work, ${ }^{7}$ Euler's theorem on rotations was used to perform rapid reorientations using reaction jets. This scheme for rapid reorientation required near-maximum jet torquing.

Flexible spacecraft slewing problems, like their rigid body counterparts, have also received considerable attention. In most of the published work the single-axis maneuver problem has been solved by minimizing a cost functional involving quadratic penalties on control effort, deformations, and deformation rates. ${ }^{19,22,24-27,32}$ It is possible to obtain feedback control strategies in these situations. An integrated structure and control optimization was carried out for rotational maneuvers minimizing a quadratic cost functional by selecting feedback control and various structural parameters simultaneously, ${ }^{30,31}$ with the maneuver time being fixed. Skaar and Tucker ${ }^{28,29}$ used a convolution integral approach to find exact open- and closedloop control solutions that bring a finite number of locations on a flexible structure to any desired final position and velocity. by minimizing a quadratic cost functional involving penalties on control effort and control effort rate during a fixed maneuver duration. Optimal open-loop controls have also been obtained that perform these slewing maneuvers while minimizing a structural excitation criterion..$^{20,21}$ In all of the above cases, the maneuvers considered were planar, and their dynamics were linear. Single-axis maneuvers including the effects of kinematic nonlinearities have also received some attention. ${ }^{23}$ Here, optimal open- and closed-loop controls were obtained by minimizing a quadratic performance index for a fixed maneuver time. The resulting nonlinear two-point boundary value problem was solved using continuation methods. A slewing control experiment has also been carried out to test the linear optimal control applied to single-axis slews of a flexible structure. ${ }^{34}$ In all of these published works the optimal control time histories were found to be continuous.

Slewing problems using on-off type actuation have recently begun to receive attention. In a recent account, ${ }^{37}$ an optimal open-loop control is obtained for a fixed maneuver time where the intermediate switching time is selected to minimize the post-maneuver energy. Near-optimal maneuvers to perform these retargetings, which minimize a weighted combination of maneuver time and fuel consumption, were also obtained recently. ${ }^{35,36}$ Thompson et al. ${ }^{38}$ have solved the planar rest-torest slewing problem by shaping the control input profiles. The resulting control is not bang-bang, and the maneuvers thus performed are not minimum time. All of the above have dealt with planar sewings. Monaco and Stronelli ${ }^{39}$ suggest a steadystate feedback control strategy for simultaneous three-axis slews, utilizing reaction wheels and on-off controls, which brings the system state to the desired final state. All of these accounts, however, stop short of obtaining a time optimal control, using on-off type actuation only, which minimizes maneuver time.

In the present work we examine the minimum time, planar, rest-to-rest rotational maneuvers of a flexible spacecraft. All controls are assumed to be magnitude limited. The spacecraft is modeled as a linear, elastic, undamped, nongyroscopic system. Time optimal control histories, which bring the rigid body mode and a finite number of vibrational modes to rest at the final time, are obtained. The system is assumed to be in a state of rest initially. The control histories are found to be bangbang with multiple switches:

The original results of this work are that:

1). The optimal control history has a time symmetry property.

2) The optimal switching times, optimal final time, and the costates at midmaneuver satisfy a system of nonlinear algebraic equations.

3) The pointing error resulting from spillover into uncontrolled models is quantified.

Based on these observations the numerical optimization problem can be reduced to a problem of solving a set of algebraic equations for only one-half of the switching times and the final time. In this work a homotopy method is used to obtain solutions.

It should be pointed out that the results obtained here are applicable to all linear, elastic, undamped, nongyroscopic mechanical systems possessing one rigid body mode and a finite number of undamped elastic modes, in rotation or translation.

To outline briefly the remainder of this paper, we formulate the time optimal control problem in Sec. II and give a specific physical example that conforms to the dynamical model described there. Next, in Sec. III, we present the important properties of the time optimal control. In Sec. IV we outline a homotopy approach that can be used to obtain solutions to the resulting set of nonlinear algebraic equations. In Sec. V we examine in detail some example maneuvers. We conclude by summarizing the original contributions and limitations of this work, and we mention future research directions.

\section{Formulation of the Time-Optimal Control Problem}

Consider the linearized rotational dynamics of a flexible spacecraft where control inputs are used to actively control the rigid body mode and flexible modes. Consider a rigid central body, as in Fig. 1, to which $N(N \geq 2)$ identical flexible appendages are attached. The following assumptions are made to obtain a mathematical model:

1) The appendages displacements, slopes, and central body rotation rates remain small.

2) The appendages are inextensible.

3) Appendage displacements are restricted to a plane orthogonal to the central body's axis of rotation.

4) The deformations of the appendages relative to their undeformed shapes are identical and antisymmetric; see Fig. 1.

5) No structural damping is present.

6) The appendages are rigidly attached to the central body with uniform spacing between them.

The spacecraft is to be controlled by a single torque actuator located on the central body and $m$ torques located at identical locations on each of the $N$ appendages (see Fig. 1). The extension of this formulation to a system controlled by force actuators or a combination of torque and force actuators is straightforward and results in a similar model.

Let $\theta(t)$ be the angular displacement of the central body, $y(x, t)$ the lateral displacement of the flexible appendages at $x$, the distance along the appendage measured from the ap- 


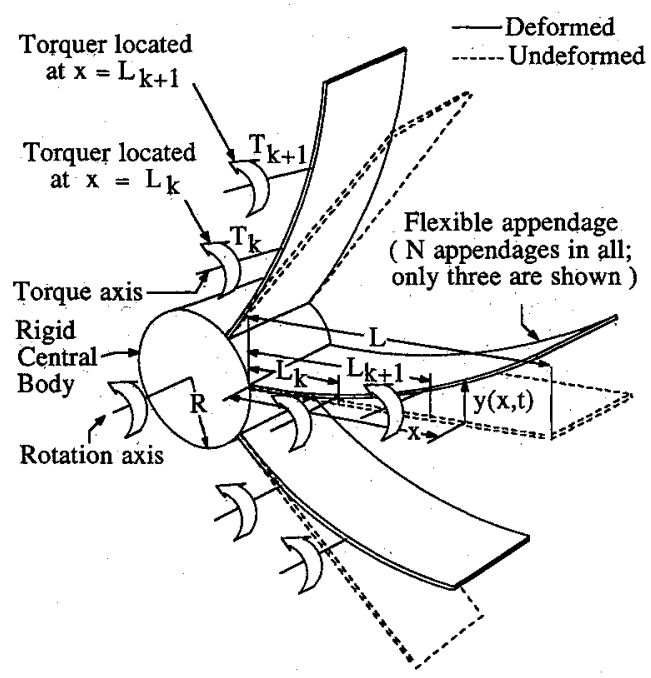

Fig. 1. Spacecraft configuration.

pendage root, $0 \leq x \leq L$; where $L$ is the overall length of one appendage. Let $Q_{\theta}$ and $Q_{y}$ be the generalized forces associated with $\theta(t)$ and $y$, respectively. Let $J^{*}$ be the rotational inertia of the undeformed vehicle about the axis of rotation, $R$ the radius of the central body (which is assumed cylindrical, with its axis of symmetry pointing along the axis of rotation), $E I(x)$ the appendage material stiffness and $\rho(x)$ be the appendage material density, respectively. Here []$_{t}[]_{x},[]_{x x}$ denote first partial time derivative, first and second partial spatial derivatives, respectively, of the quantity within parentheses and [ ]' denotes transpose.

Application of Hamilton's principle ${ }^{40}$ results in the following coupled linear partial and ordinary differential equations and the associated boundary conditions:

$$
\begin{gathered}
J^{*} \ddot{\theta}+N \int_{0}^{L} \rho(x)(R+x) y_{t t} \mathrm{~d} x=Q_{\theta} \\
N\left\{\left[E I(x) y(x, t)_{x x}\right]_{x x}+\rho(x)\left[y(x, t)_{t t}+(R+x) \ddot{\theta}\right]\right\}=Q_{y} \\
\left\{\left[E I(x) y_{x x}\right]_{x} \delta y\right\}_{(x=L)}-\left\{\left[E I(x) y_{x x}\right]_{x} \delta y\right\}_{(x=0)}=0 \\
{\left[E I(x) y_{x x} \delta y_{x}\right]_{(x=L)}-\left[E I(x) y_{x x} \delta y_{x}\right]_{(x=0)}=0}
\end{gathered}
$$

Let the appendage lateral displacement be expressed as $y(x, t)=\Sigma_{i=1}^{n} \phi_{i}(x) q_{i}(t)$; where $\phi_{i}(x)$ and $q_{i}(t)$ are the assumed mode shape and the corresponding generalized coordinate respectively $(i=1,2,3, \ldots, n)$, and $n$ is the number of vibrational modes retained in the control model. Further, define the following quantities:

$$
\begin{aligned}
& \phi=\left[\begin{array}{lllll}
\phi_{1}(x) & \phi_{2}(x) & \phi_{3}(x) & \cdots & \phi_{n}(x)
\end{array}\right] \\
& \phi_{x x}=\left\{\begin{array}{lllll}
{\left[\phi_{1}(x)\right]_{x x}} & {\left[\phi_{2}(x)\right]_{x x}} & {\left[\phi_{3}(x)\right]_{x x}} & \cdots & {\left[\phi_{n}(x)\right]_{x x}}
\end{array}\right\} \\
& q=\left[\begin{array}{lllll}
q_{1}(t) & q_{2}(t) & q_{3}(t) & \cdots & q_{n}(t)
\end{array}\right] \\
& \boldsymbol{m}=N \int_{0}^{L} \rho(x)(R+x) \boldsymbol{\phi} \mathrm{d} x \\
& \mathbf{M}=\int_{0}^{L} \rho(x) \phi \phi^{\prime} \mathrm{d} x \\
& \mathbf{K}=N \int_{0}^{L} E I(x) \phi_{x x} \phi_{x x}^{\prime} \mathrm{d} x
\end{aligned}
$$

If $T$ is the torque applied by the torquer located on the central body and $T_{j}$ is the torque applied by the torquer located at $x=L_{j}$ on an appendage, where $j=1,2, \ldots, m$ (see Fig. 1), then it can be shown that the generalized forces are

$$
Q_{\theta}=T+N 1^{\prime} \tau, \quad Q_{j i}=N T_{j}\left\{\left[\phi_{i}(x)\right]_{x}\right\}_{(x=L j)}
$$

where $\tau=\left(\begin{array}{llllll}T_{1} & T_{2} & T_{3} & \ldots & T_{m}\end{array}\right)^{\prime}$ and $\mathbf{1}=\left(\begin{array}{lllll}1 & 1 & 1 & \ldots & 1\end{array}\right)^{\prime}$ is a vector of appropriate dimension. The quantities defined above, when used in Eqs. (1) and (2), result in the following coupled linear ordinary differential equations:

$$
\begin{aligned}
& J^{*} \ddot{\theta}+\boldsymbol{m}^{\prime} \ddot{\boldsymbol{q}}=T+N \mathbf{1}^{\prime} \tau \\
& \mathbf{M} \ddot{q}+\mathbf{K} \boldsymbol{q}+\boldsymbol{m} \ddot{\theta}=\Phi_{x} \tau
\end{aligned}
$$

where the $i j$ th element of $\boldsymbol{\Phi}_{x}$ is $N\left\{\left[\phi_{i}(x)\right]_{x}\right\}_{(x=L i)}$. Using Eq. (6) in Eq. (7) we obtain:

$$
\left[\mathbf{M}-\left(1 / J^{*}\right) \boldsymbol{m} \boldsymbol{m}^{\prime}\right] \ddot{\boldsymbol{q}}+\mathbf{K} \dot{q}=-\left(T / J^{*}\right) \boldsymbol{m}+\left[\boldsymbol{\Phi}_{x}-\left(N / J^{*}\right) \boldsymbol{m} \mathbf{1}^{\prime}\right] \tau
$$

Introducing a coordinate transformation $\boldsymbol{q}=\mathbf{U} \boldsymbol{\eta}$, where $\mathbf{U}$ is a normalized modal matrix such that $\mathbf{U}^{\prime}\left[\mathbf{M}-\left(1 / J^{*}\right) \mathrm{mm}^{\prime}\right]$ $\mathbf{U}=\boldsymbol{I}$ and $\mathbf{U}^{\prime} \mathbf{K} \mathbf{U}=\mathbf{\Omega}^{2}$ [where $\mathbf{I}$ is a unit matrix and $\mathbf{\Omega}^{2}=$ $\operatorname{diag}\left(\omega_{i}^{2}\right)$, and $\left.i=1,2,3, \ldots, n\right]$, Eq. (8) is transformed into

$$
\ddot{\boldsymbol{\eta}}+\boldsymbol{\Omega}^{2} \boldsymbol{\eta}=-\left(T / J^{*}\right) \mathbf{U}^{\prime} \boldsymbol{m}+\mathbf{U}^{\prime}\left\{\boldsymbol{\Phi}_{\boldsymbol{x}}-\left(N / J^{*}\right) \boldsymbol{m} \mathbf{1}^{\prime}\right\} \boldsymbol{\tau}
$$

Finally we define the following state and control variables:

$x_{1}=\theta+\left(1 / J^{*}\right) m^{\prime} \boldsymbol{q}, \quad x_{2}=\dot{x}_{1}, x_{3}^{i}=\eta_{i}, x_{4}^{i}=\dot{\eta}_{i} / \omega_{i} ; \quad i=1,2, \ldots, n$

$$
u_{0}=T / J^{*}, \quad u_{j}=\left(N / J^{*}\right) T_{j}, \quad j=1,2, \ldots, m
$$

and the parameters:

$$
\beta_{0}^{i}=-\left(\boldsymbol{\Omega}^{-1} \mathbf{U}^{\prime} \boldsymbol{m}\right)_{i}, \quad i=1,2, \ldots, n
$$

$\beta_{j}^{i}=\left[\mathbf{\Omega}^{-1} \mathbf{U}^{\prime}\left\{\left(J^{*} / N\right) \boldsymbol{\Phi}_{\boldsymbol{x}}-\boldsymbol{m} \mathbf{1}^{\prime}\right\}\right]_{i j}, \quad i=1,2, \ldots, n ; j=1,2, \ldots, m$

here [ ] $]^{-1}$ denotes matrix inverse, [ $]_{i}$ and []$_{i j}$ stand for the $i$ th and $i j$ th elements of the argument [ ], respectively.

Using Eqs. (10-13) in Eqs. (6) and (9), the following state space description of the plant dynamics are obtained:

$$
\begin{gathered}
\dot{x}_{1}=x_{2} \\
\dot{x}_{2}=\sum_{j=0}^{m} u_{j} \\
\dot{x}_{3}^{i}=\omega_{i} x_{4}^{i}, \quad i=1,2,3, \ldots, n \\
\dot{x}_{4}^{i}=-\omega_{i} x_{3}^{i}+\sum_{j=0}^{m} \beta_{j}^{i} u_{j}, \quad i=1,2,3, \ldots, n .
\end{gathered}
$$

Defining the state vector as $\boldsymbol{x}=\left(x_{1}, x_{2}, x_{3}^{1}, x_{4}^{1}, x_{3}^{2}, x_{4}^{2}, \ldots, x_{3}^{n}, x_{4}^{n}\right)^{\prime}$ and the control vector as $\boldsymbol{u}=\left(u_{0}, u_{1}, u_{2}, \ldots, u_{m}\right)^{\prime}$, we obtain the state representation of the control model

$$
\dot{\boldsymbol{x}}(t)=\mathbf{A} \boldsymbol{x}(t)+\mathbf{B} u(t)
$$

where $\mathbf{A}=$ block diag $\left[\mathbf{A}_{i}\right], \mathbf{B}=$ block $\operatorname{col}\left[\mathbf{B}_{i}\right]$ and

$$
\begin{gathered}
\mathbf{A}_{i}=\left[\begin{array}{ll}
0 & 1 \\
0 & 0
\end{array}\right], i=0 \\
\mathbf{A}_{i}=\left[\begin{array}{cc}
0 & \omega_{i} \\
-\omega_{i} & 0
\end{array}\right], i=1,2, \ldots, n \\
\mathbf{B}_{i}=\left[\begin{array}{cccc}
0 & 0 & \cdots & 0 \\
\beta_{0}^{i} & \beta_{1}^{i} & \cdots & \beta_{m}^{i}
\end{array}\right], i=0,1,2, \ldots, n
\end{gathered}
$$


where $\beta_{j}^{0}=1 ; j=0,1,2, \ldots, m$. The pair $(\mathbf{A}, \mathbf{B})$ is assumed to be normal. 44

The following constraints are imposed on the $m+1$ controllers:

$$
\left|u_{j}\right| \leq U_{j}, \quad j=0,1,2, \ldots, m
$$

The boundary conditions to be satisfied are:

$$
\boldsymbol{x}\left(-t_{f} / 2\right)=\left(-\theta_{f} / 2,0,0,0, \ldots, 0\right)^{\prime}, \quad \boldsymbol{x}\left(t_{f} / 2\right)=\left(\theta_{f} / 2,0,0,0, \ldots, 0\right)^{\prime}
$$

where $\theta_{f}$ is the total slew angle. It is assumed, without loss of generality, that the time interval over which the controls are active is $\left[-t_{f} / 2, t_{f} / 2\right]$. Outside of this interval all controls are turned off, i.e. $\boldsymbol{u}(t)=\mathbf{0}$. We wish to find the optimal control vector $u(t)^{*}$ that minimizes $t_{f}$ while satisfying Eqs. (18-22).

\section{Properties of the Time-Optimal Control}

Pontryagin's maximum principle ${ }^{41}$ is used to characterize the time-optimal control vector $u(t)^{*}$. It has been assumed, without loss of generality, that the maneuver is centered about time $t=0$. Exactly $n$ modes are suppressed at the final time. We use the Hamiltonian $\mathbb{H}=1+\boldsymbol{p}(t)^{\prime}[\mathbf{A x}(t)+\mathbf{B} u(t)]$, where $\boldsymbol{p}(t)=$ $\left[p^{0}(t), q^{0}(t), p^{1}(t), q^{\mathrm{l}}(t), \ldots, p^{n}(t), q^{n}(t)\right]^{\prime}$ is the costate vector. The necessary (and in this case sufficient) conditions for optimality, along with Eqs. (18-22), are:

$$
\dot{\boldsymbol{p}}(t)=-\mathbf{A}^{\prime} \boldsymbol{p}(t)
$$

where $t \in\left[-t_{f}^{*} / 2, t_{f}^{*} / 2\right]$,

$$
u_{j}(t)^{*}=-U_{j} \operatorname{sgn}\left[\sum_{i=0}^{n} \beta_{j}^{i} q^{i}(t)\right]
$$

where $j=0,1,2,3, \ldots, m$ and $t \in\left[-t_{f}^{*} / 2, t_{f}^{*} / 2\right]$

$$
\mathrm{H}\left(t_{f}^{*} / 2\right)+\mathrm{H}\left(-t_{f}^{*} / 2\right)=0
$$

where $\operatorname{sgn}(x)=1$ if $x>0$ and $\operatorname{sgn}(x)=-1$ if $x<0$, is the signum function. Here, Eq. (25) also implies that $\mathbb{H}(t) \equiv 0$ where $t \in\left[-t_{f}^{*} / 2, t_{f}^{*} / 2\right]$.

It is also straightfoward to show ${ }^{42}$ that the optimal control exists, is unique, and is of the bang-bang variety with, possibly, multiple switches in each control variable. The normality of $(\mathbf{A}, \mathbf{B})$ prohibits the existence of singular arcs.

Theorem 1: For the optimal control problem defined by Eqs. (18-22), the optimal control $u(t)^{*}$ is an odd function of time, i.e., $u(t)^{*}=-u(-t)^{*},-t_{f}^{*} / 2 \leq t \leq t_{f}^{*} / 2$, where $t_{f}^{*}$ is the optimal maneuver time.

Corollary 1: For the optimal control problem defined by Eqs (18-22), the optimal costate vector satisfying Eq. (23) is such that:

$$
p(0)=\left(p_{0}^{0}, 0, p_{0}^{1}, 0, \ldots, p_{0}^{n}, 0\right)^{\prime}
$$

Theorem 1 implies that each control variable must switch an odd number of times. They do not necessarily switch at the same times, but all switch synchronously at time $t=0$. Suppose that the $j$ th optimal control variable, $u_{j}(t)^{*}$, switches $2 k^{j}+1$ times, where $j=0,1,2, \ldots, m$; and define $v=\Sigma_{j=0}^{m} k^{j}$. These switches must occur at times $-t_{k^{j}}^{j},-t_{k^{j}-1}^{j}, \ldots,-t_{1}^{j}, 0$, $t_{1}^{j}, \ldots, t_{k^{j}-1}^{j}, t_{k}^{j}$; where these switching instants satisfy

$$
0<t_{1}^{j}<t_{2}^{j}<\cdots<t_{k^{j}-1}^{j}<t_{k^{j}}^{j}<t_{f} / 2, \quad j=0,1,2, \ldots, m
$$

These switching instants for each control input are distinct because coincidence of two switching instants would imply that the respective control does not switch at such a time. Let

$$
\alpha_{j}=\operatorname{sgn}\left[u_{j}(t)\right], \quad-t_{f} / 2<t<-t_{k^{i}}^{j}, \quad j=0,1,2, \ldots, m
$$

represent the initial sign of $u_{j}(t)$. Because $u_{j}(t)$ changes sign at

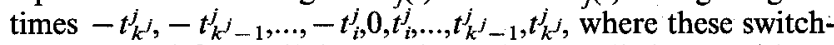
ing times satisfy Eq. (27), Eqs. (14-17) are easily integrated. To satisfy the boundary conditions (22), the following algebraic equations, which govern the switching times and the final time, are obtained:

$$
\begin{aligned}
& \sum_{j=0}^{m} \alpha_{j} U_{j}\left[\left(t_{f} / 2\right)^{2}-2\left(t_{k^{j}}^{j}\right)^{2}+2\left(t_{k^{j}-1}^{j^{j}}\right)^{2}\right. \\
& \left.-\cdots+2(-1)^{k^{j}}\left(t_{1}^{j}\right)^{2}\right]=\theta_{f} \\
& \sum_{j=0}^{m} \alpha_{j} \beta_{j}^{i} U_{j}\left[\cos \left(\omega_{i} t_{f} / 2\right)-2 \cos \left(\omega_{i} t_{k^{j}}^{j}\right)\right. \\
& \left.+\cdots+2(-1)^{k^{j}} \cos \left(\omega_{i} t_{1}^{j}\right)+(-1)^{k^{j}+1}\right]=0
\end{aligned}
$$

where $i=1,2, \ldots, n$.

Moreover, Eqs. (26) and (23) allow a closed-form computation of the switching function of each control variable. Requiring that these switching functions vanish at their respective switching times and satisfaction of Eq. (25) implies:

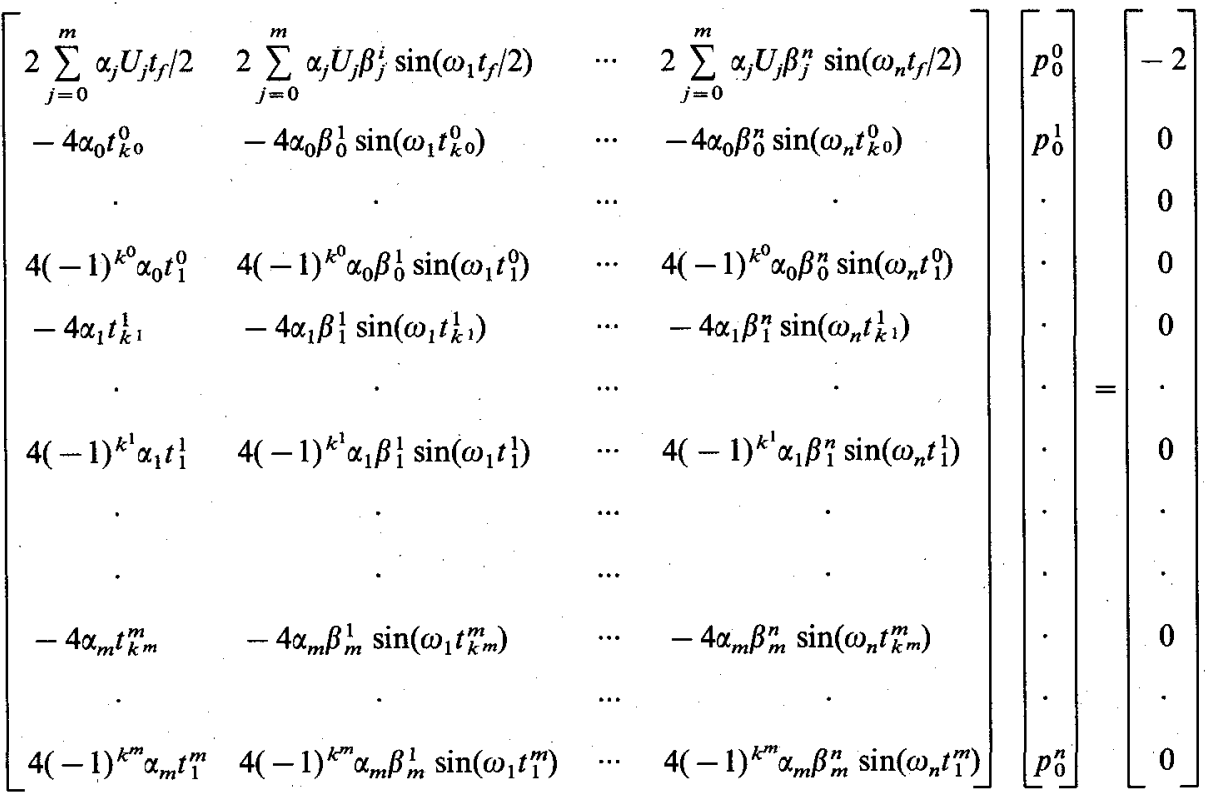


Equations (29-31) represent a system of $n+v+2$ nonlinear algebraic equations in $n+v+2$ unknowns that are as follows: $v$ switching times $t_{r}^{j}, 1 \leq r \leq k^{j}, j=0,1, \ldots, m$; the final time $t_{f} / 2$; and the $n+1$ costates at midmaneuver $p_{0}^{0}, p_{0}^{l}, \ldots, p_{0}^{n}$. Because Eqs. (29-31) have been obtained from Eqs. (18-22), they are necessary conditions for optimality. In addition, Eqs. (29-31) are also sufficient provided that

$$
\boldsymbol{p}^{\prime}(t) \boldsymbol{b}_{j} \neq 0, \quad t \neq t_{r}^{j}
$$

where $1 \leq r \leq k^{j}, j=0,1,2, \ldots, m$, and $0<t<t_{f} / 2$. Also, where $p(t) \in \mathbb{R}^{2 n+2}$ is defined by Eqs. (26) and (23), and $b_{j}$ is the $j$ th column of $\mathbf{B}$ in Eq. (18), $0 \leq j \leq m$. This implies that on the interval $\left(0, t_{f} / 2\right)$, the only zeros of the $j$ th candidate switching function are the candidate switching times $t_{r}^{j}, 1 \leq r \leq k^{j}$, and $j=0,1, \ldots, m$.

Theorem 2: Suppose that for some integers $k^{j}, 0 \leq j \leq m$, Eqs. (29-31) admit a solution $\left(t_{r}^{j}, 1 \leq r \leq k^{j}, 0 \leq j \leq m ; t_{f} / 2\right.$; $\left.p_{0}^{i}, 0 \leq i \leq n\right)$ satisfying the inequalities of Eqs. (27) and (32). Then 1) the $j$ th component of the optimal control solving problem (18-22) switches exactly $2 k^{j}+1$ times at instants $-t_{k^{j}}^{j},-t_{k^{j}-1}^{j_{1}}, \ldots,-t_{1}^{j}, 0, t_{1}^{j}, \ldots, t_{k^{j}-1}^{j}, t_{k^{j}}^{j}$; and 2) the optimal maneuver time of problem (18-22) is exactly $t_{f}$.

We notice that if $v=n$, Eqs. (29) and (30) are a set of $n+1$ equations with $n+1$ unknowns. Under a mild additional assumption, the solution of Eqs. (29) and (30) completely characterizes the optimal control. We have the following corollary.

Corollary 2: Suppose that integers $k^{j}, 0 \leq j \leq m$ satisfy $\sum_{j=0}^{m} k^{j}=n$. Also, assume that Eqs. (29) and (30) admit a solution $\left(t_{r}^{j}, 1 \leq r \leq k^{j}, 0 \leq j \leq m ; t_{f} / 2\right)$ that is regular ${ }^{43}$ and satisfies Eqs. (27), (31), and (32). Then the claims of Theorem 2 are true.

Equations (29-31) form the basis of a numerical algorithm to solve the optimal control problem (18-22). One potential difficulty with these equations is that $\alpha_{j}, k^{j}$, where $0 \leq j \leq m$ are not known a priori. In our examples we have chosen $m=0$, corresponding to a single control variable. We have noticed that in most cases $v=n$ and we have extensively made use of Corollary 2. The case $v<n$ is exceptional but can occur if modal frequencies are rational multiples of each other. The case $v>n$ can also occur, but seems to be restricted to some particular ranges of the parameter $\left(\theta_{f} / U_{0}\right) \omega_{1}^{2}$. To obtain the optimal switching times and the final time we proceed as follows: We suppose that $v=n$ and use Corollary 2 to determine if the solution is optimal. If Eqs. (29) and (30) do not admit a regular solution, we explore the situation $v \neq n$ by increasing or decreasing $v$ and use Theorem 2 to determine whether a candidate solution is indeed optimal; if it is, then it is the only solution to the optimal control problem, because Eqs. (29-32) are both necessary and sufficient for optimality.

\section{Control Spillover}

The optimal control characterized in Sec. II is based on a control model which has $n$ flexible modes. This control law is now applied to an evaluation model, of the same form as Eqs. (14-17), which has $M$ flexible modes $(M \geq n)$ in addition to the rigid body mode. We introduce the following notation:

$$
\boldsymbol{x}^{i}(t)=\left[x_{3}^{i}(t), x_{4}^{i}(t)\right]^{\prime}
$$

where $i=n+1, n+2, \ldots, M$ and

$$
\begin{gathered}
\boldsymbol{\sigma}=\mathbf{U}^{\prime} \boldsymbol{m} / J^{*}=\left(\sigma_{1}, \sigma_{2}, \ldots, \sigma_{M}\right)^{\prime} \\
\theta_{\varepsilon}(t)=\theta(t)-\theta_{f} / 2
\end{gathered}
$$

Consider the effect of control spillover on the $M-n$ residual modes whose dynamics are given by Eqs. (16) and (17). In this analysis, assume that the control is given by

$$
\begin{aligned}
\boldsymbol{u}(t) & =\boldsymbol{u}(t)^{*}, & & t \in\left[-t_{f}^{*} / 2, t_{f}^{*} / 2\right] \\
& =\mathbf{0}, & & t \notin\left[-t_{f}^{*} / 2, t_{f}^{*} / 2\right]
\end{aligned}
$$

The spillover results in free vibration after the maneuver has been completed. This spillover effect is now characterized. Equations (16) and (17), when integrated, result in

$$
\boldsymbol{x}^{i}(t)=\exp \left(\mathbf{A}_{i} t\right) \int_{-t_{f}^{*} / 2}^{t_{f}^{*} / 2} \exp \left(-\mathbf{A}_{i} t\right) \mathbf{B}_{i} \boldsymbol{u}(t)^{*} \mathrm{~d} t
$$

where $t \geq t_{f}^{*} / 2, i=n+1, n+2, \ldots, M$. Therefore

$$
\boldsymbol{x}^{i}(t)=\left[\begin{array}{c}
-2 \sum_{j=0}^{m} \beta_{j}^{i} S_{i j} \cos \left(\omega_{i} t\right) \\
2 \sum_{j=0}^{m} \beta_{j}^{i} S_{i j} \sin \left(\omega_{i} t\right)
\end{array}\right], \quad t \geq t_{f}^{*} / 2
$$

where the constants

$$
\begin{gathered}
S_{i j}=\int_{0}^{t_{f}^{*} / 2} u_{j}(t)^{*} \sin \left(\omega_{i} t\right) \mathrm{d} t \\
S_{i j}=\alpha_{j}\left(U_{j} / \omega_{i}\right)\left[\cos \left(\omega_{i} t_{f}^{*} / 2\right)-2 \cos \left(\omega_{i} t_{k^{*}}^{i}\right)\right. \\
+\cdots+2(-1)^{k j} \cos \left(\omega_{i} t_{1}^{i *}\right)+(-1)^{k^{j}+1}
\end{gathered}
$$

where $i=n+1, n+2, \ldots, M$ and $j=0,1,2, \ldots, m$. Note that $\Sigma_{j=0}^{m} \beta_{j}^{i} S_{i j}=0 \forall i \leq n$.

The constant residual energy, after completion of the maneuver, is defined as $E_{r}=(1 / 2) \sum_{i=n+1}^{M}\left[\omega_{i}^{2} x^{i}\left(t_{f}^{*} / 2\right)^{\prime} x^{i}\left(t_{f}^{*} / 2\right)\right]$ and can be expressed as

$$
E_{r}=2 \sum_{i=n+1}^{M}\left[\omega_{i}^{2}\left(\sum_{j=0}^{m} \beta_{j}^{i} S_{i j}\right)^{2}\right]
$$

Spillover into the residual modes results in an attitude error for the rigid central body. A bound on this attitude error can be obtained. Using Eq. (10) we obtain

$$
\theta_{\varepsilon}(t)=2 \sum_{i=n+1}^{M}\left[\sigma_{i} \cos \left(\omega_{i} t\right)\left(\sum_{j=0}^{m} \beta_{j}^{i} S_{i j}\right)\right]
$$

where $t \geq t_{f}^{*} / 2$. Using Eqs. (35) and (36) in Eq. (38), two upper bounds on $\left|\theta_{\varepsilon}(t)\right|$ are obtained. Using Eq. (35) we obtain

$$
\left|\theta_{\varepsilon}(t)\right| \leq t_{f}^{*} \sum_{i=n+1}^{M}\left|\left[\sigma_{i}\left(\sum_{j=0}^{m} \beta_{j}^{i} U_{j}\right)\right]\right|
$$

where $t \geq t_{f}^{*} / 2$, and using Eq. (36) we obtain a separate bound:

$$
\left|\theta_{\varepsilon}(t)\right| \leq 2 \sum_{i=n+1}^{M}\left|\left\{\left(\sigma_{i} / \omega_{i}\right)\left[\sum_{j=0}^{m} \alpha_{j} \beta_{j}^{i} U_{j}\left(2+2 k^{j}\right)\right]\right\}\right|
$$

where $t \geq t_{f}^{*} / 2$. The bound in Eq. (40) is tighter than the bound in Eq. (39) if:

$$
\sum_{j=0}^{m} \beta_{j}^{i} U_{j} \omega_{i} t_{f}^{*} / 2>\sum_{j=0}^{m}\left[\alpha_{j} \beta_{j}^{i} U_{j}\left(2+2 k^{j}\right)\right]
$$

where $i=n+1, n+2, \ldots, M$. These expressions are further simplified when we examine the scalar control case.

\section{Scalar Control Case}

Here we assume that only one control input is used to control the maneuver. This assumption, in terms of the assumptions about the physical models (Fig. 1) means that the $N$ torque actuators, one on each appendage with identical loca- 
tions on the appendages, taken together represent one control input. We can also consider a spacecraft controlled by only one torque actuator located on the rigid central body as another special scalar control case. Equations (29) and (30) become:

$$
\begin{gathered}
\alpha\left[\left(t_{f} / 2\right)^{2}-2\left(t_{k}\right)^{2}+2\left(t_{k-1}\right)^{2}-\cdots+2(-1)^{k}\left(t_{1}\right)^{2}\right]=\theta_{f} / U_{0} \\
\cos \left(\omega_{i} t_{f} / 2\right)-2 \cos \left(\omega_{i} t_{k}\right)+2 \cos \left(\omega_{i} t_{k-1}\right)-\cdots+2(-1)^{k} \cos \left(\omega_{i} t_{1}\right)+(-1)^{k+1}=0
\end{gathered}
$$

where $i=1,2, \ldots, n$. Equation (31) can be written as:

$$
\left[\begin{array}{cccc}
2 \alpha U_{0} t_{f} / 2 & 2 \alpha U_{0} \sin \left(\omega_{1} t_{f} / 2\right) & \cdots & 2 \alpha U_{0} \sin \left(\omega_{n} t_{f} / 2\right) \\
-4 \alpha t_{k} & -4 \alpha \sin \left(\omega_{1} t_{k}\right) & \cdots & -4 \alpha \sin \left(\omega_{n} t_{k}\right) \\
\cdot & \cdot & \cdots & \cdot \\
4(-1)^{k} \alpha t_{1} & 4(-1)^{k} \alpha \sin \left(\omega_{1} t_{1}\right) & \cdots & 4(-1)^{k} \alpha \sin \left(\omega_{n} t_{1}\right)
\end{array}\right]\left[\begin{array}{c}
p_{0}^{0} \\
\beta_{j}^{1} p_{0}^{1} \\
\cdot \\
\beta_{j}^{n} p_{0}^{n}
\end{array}\right]=\left[\begin{array}{c}
-2 \\
0 \\
\cdot \\
0
\end{array}\right]
$$

where we have used $\alpha=\alpha_{j}$ and $\beta_{j}^{i}, i=0,1,2, \ldots, n$ are defined in Eqs. (12) and (13). (The only difference is that the index $j$ is fixed here for the given actuator location; e.g., $j=0$ for the actuator location on the rigid central body.) Satisfaction of Eq. (32) implies that

$$
p_{0}^{0} t+\sum_{i=1}^{n} \beta_{j}^{i} p_{0}^{i} \sin \left(\omega_{i} t\right) \neq 0
$$

where $t \neq t_{r}, 0 \leq t \leq t_{f} / 2$, and $r=1,2, \ldots, k$.

Costates at midmaneuver, and $\beta_{j}^{i}$ and $i=1,2, \ldots, n$ appear here as $p_{0}^{0}, \beta_{j}^{1} p_{0}^{1}, \ldots, \beta_{j}^{n} p_{0}^{n}$. It is evident that for the given optimal control problem with a scalar input, if only $\beta_{j}^{i}$ and $i=1,2, \ldots, n$ are changed through a change in the actuator location, so long as $\beta_{j}^{i} \neq 0$ and $i=1,2, \ldots, n$, (which is prohibited from normality considerations), it is always possible to rescale $p_{0}^{i}, i=1,2, \ldots n$ (such that $\beta_{j}^{i} p_{0}^{i}$ and $i=1,2, \ldots, n$ remain invariant) so that Eqs. (44) and (45) are still satisfied for the same values of switching and final times. We therefore observe the following proposition:

Proposition: As indicated above, the time-optimal control history of the system [Eqs. (18-22)], where control is effected using only one control input, is independent of actuatory location. It only depends on the modal frequencies, $\omega_{i}$ and $i=1,2, \ldots, n$, and the parameter $\theta_{f} / U_{0}$.

The preceding proposition would enable us to optimize the actuatory location such that some structural excitation criterion is minimized, without effecting the optimal maneuver time.

It has been observed upon solving Eqs. (42) and (43) that for $\left(\theta_{f} / U_{0}\right) \omega_{1}^{2} \gg 1$, the maneuver time is not significantly different from the time needed to maneuver an equivalent rigid body in a time-optimal fashion. This is apparently true regardless of the number of actively controlled modes. Active suppression of additional modes at the final time results in different optimal control history without a significant increase in maneuver time, provided that $\left(\theta_{f} / U_{0}\right) \omega_{1}^{2}$ is sufficiently large. Assuming $\left(\theta_{f} / U_{0}\right) \omega_{1}^{2} \gg 1$, the following serves as a good approximation of the optimal maneuver time:

$$
t_{f}^{*} \cong 4\left(\mathbb{P} / \omega_{1}\right)\left\{1-[\sin (\mathbb{P}) / \mathbb{P}]^{2}\right\}^{-0.5}
$$

where $\mathbb{P}=0.5\left[\left(\theta_{f} / U_{0}\right) \omega_{1}^{2}\right]^{0.5}$. Here we note that the optimal slewing time for an equivalent rigid body is $4\left(\mathbb{P} / \omega_{1}\right)$.

The approximations to the post-maneuver energy and the attitude error of the central body are obtained using Eqs. (37) and (38), respectively. These are:

$$
E_{r}=2 \sum_{i=n+1}^{M}\left(\omega_{i} \beta_{j}^{i} S_{i}\right)^{2}
$$

$$
\left|\theta_{s}(t)\right| \leq 2 \sum_{i=n+1}^{M}\left|\left(\sigma_{i} \beta_{j}^{i} S_{i}\right)\right|, \quad t \geq t_{f}^{*} / 2
$$

Since $S_{i j}$, as defined previously, does not depend on $j$, we have used $S_{i}=S_{i j}$ where $i=n+1, n+2, \ldots, M$. Equations (39) and (40) become

$$
\begin{gathered}
\left|\theta_{\varepsilon}(t)\right| \leq U_{0} t_{f}^{*} \sum_{i=n+1}^{M}\left|\left(\sigma_{i} \beta_{j}^{i}\right)\right| \\
\left|\theta_{\varepsilon}(t)\right| \leq 4 U_{0}(n+1) \sum_{i=n+1}^{M}\left|\left(\sigma_{i} \beta_{j}^{i} / \omega_{i}\right)\right| \quad t \geq t_{f}^{*} / 2
\end{gathered}
$$

respectively, where we have supposed that the control switches of $2 n+1$ times. Once again, Eq. (50) provides a tighter bound than Eq. (49) if

$$
\omega_{i} t_{f}^{*} / 2 \geq 2(n+1)
$$

where $i=n+1, n+2, \ldots, M$.

\section{Determination of the Time-Optimal Control}

As observed in Corollary 2, the numerical optimization problem can be reduced to a set of algebraic equations in Eqs. (29) and (30). The solutions of these equations can be obtained using a homotopy scheme. ${ }^{43}$ The scheme proposed here is applicable to the scalar control case described previously. The generalization to the vector control case is straightforward. We define the following nondimensional variables:

$$
y_{i}=\omega_{1} t_{i}, \quad y_{n+1}=\omega_{1} t_{f} / 2
$$

where $i=1,2, \ldots, n$. The constant parameters are defined as

$$
r_{i}=\omega_{i} / \omega_{1} \geq 1, \quad i=1,2, \ldots, n, \quad P=\omega_{1}^{2} \theta_{f} / U_{0}
$$

Table 1 Spacecraft dimensions, appendage material, and maneuver specifications

Radius of the rigid central body, $R$

Length of one appendage, $L$

Appendage material stiffness, $E I$

Appendage material density, $\rho$

Mass of the rigid central body

Maximum torque available, $T$

Command slew angle, $\theta_{f}$

Total rotational inertia, $J^{*}$

$1.00 \mathrm{~m}$
$40.00 \mathrm{~m}$
$1500.00 \mathrm{~N}-\mathrm{m}^{2}$
$0.04096 \mathrm{~kg} / \mathrm{m}$
$400.00 \mathrm{~kg}$
$150.00 \mathrm{~N}-\mathrm{m}$
$90.00 \mathrm{deg}$
$2081.97547 \mathrm{~kg}-\mathrm{m}^{2}$


Table 2 Modal quantities

\begin{tabular}{rrrrrrrrrrr}
\hline \hline$i$ & 1. & 2 & 3 & 4 & \multicolumn{1}{c}{5} & 6 & \multicolumn{1}{c}{7} & \multicolumn{1}{c}{8} & 9 & \multicolumn{1}{c}{10} \\
\hline$\omega_{i}$ & 1.151 & 3.009 & 7.529 & 14.546 & 23.962 & 35.752 & 49.908 & 66.429 & 85.312 & 106.557 \\
$\beta_{j}^{i}$ & 98.283 & 24.404 & 3.946 & 1.102 & 0.428 & 0.203 & 0.110 & 0.065 & 0.041 & 0.028 \\
\hline
\end{tabular}

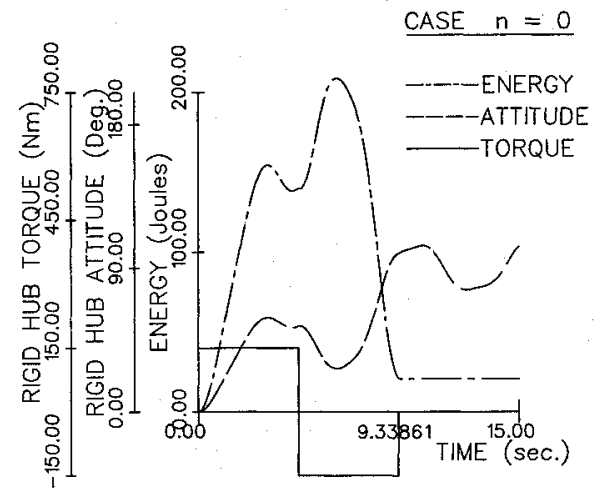

Fig. 2 The energy, rigid central body attitude and optimal control time histories for $\boldsymbol{n}=\mathbf{0}$.

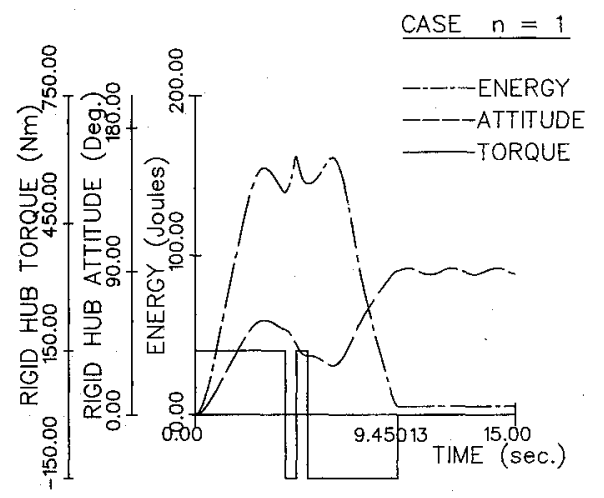

Fig. 3 The energy, rigid central body attitude and optimal control time histories for $n=1$.

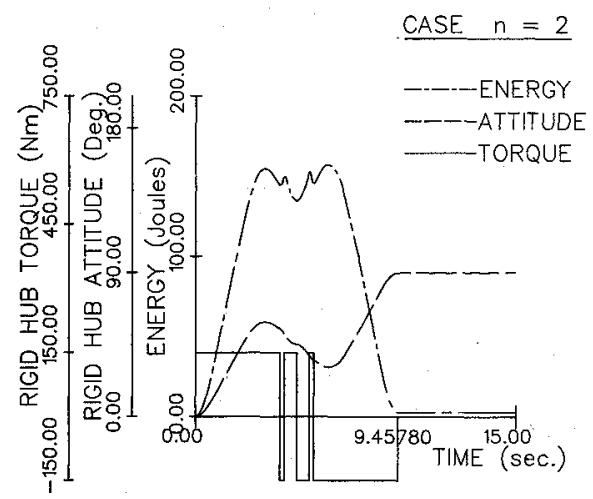

Fig. 4 The energy, rigid central body attitude and optimal control time histories for $n=2$.

We will refer to $P$ as the maneuver parameter. Equations (29) and (30) can now be rewritten as:

$$
\begin{aligned}
& F_{j}\left(y_{n+1}, y_{n}, \ldots, y_{2}, y_{1}\right)=y_{n+1}{ }^{2}+2 \sum_{i=1}^{n}(-1)^{i} y_{n-i+1}{ }^{2}-P / \alpha=0 \\
& F_{j}\left(y_{n+1}, y_{n}, \ldots, y_{2}, y_{1}\right)=\cos \left(r_{j} y_{n+1}\right) \\
& \quad+2 \sum_{i=1}^{n}(-1)^{i} \cos \left(r_{j} y_{n-i+1}\right)+(-1)^{n+1}=0
\end{aligned}
$$

Table 3 Optimal final time and switching times for the four cases $(P=28.8706)$, s

\begin{tabular}{cccccc}
\hline \hline$n$ & $t_{f} / 2$ & $t_{n}$ & $t_{n-1}$ & $t_{n-2}$ & $t_{n-3}$ \\
\hline 1 & 4.7250660 & 0.5117851 & - & - & - \\
2 & 4.7280912 & 0.7807244 & 0.5739997 & - & - \\
3 & 4.7292194 & 0.8517724 & 0.7056135 & 0.2332373 & - \\
4 & 4.7292236 & 0.8530396 & 0.7079743 & 0.2406521 & 0.0527481 \\
\hline \hline
\end{tabular}

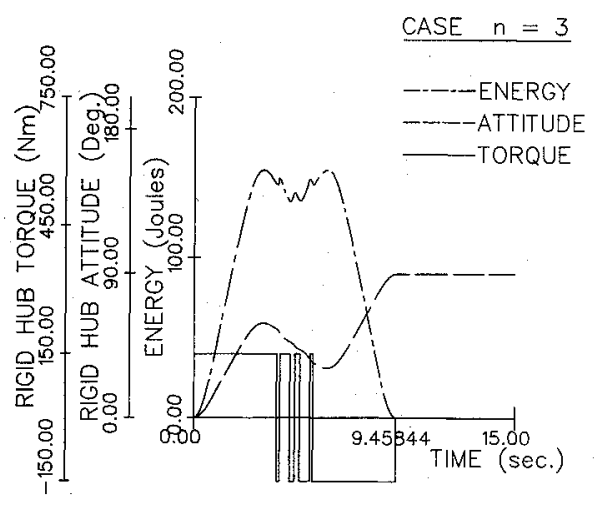

Fig. 5 The energy, rigid central body attitude and optimal control time histories for $n=3$.

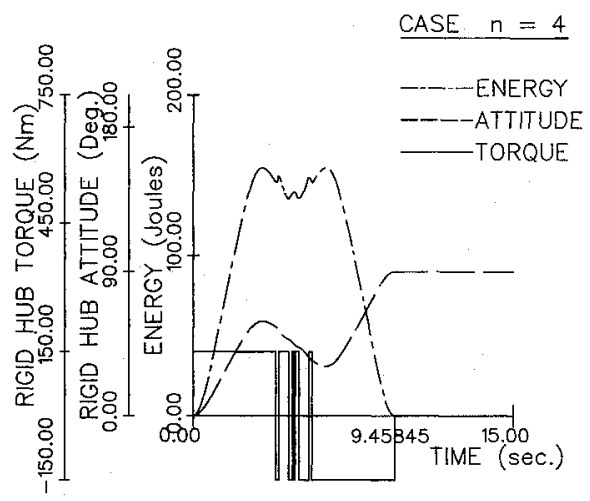

Fig. 6 The energy, rigid central body attitude and optimal control time histories for $n=4$.

where $j=1,2, \ldots, n$, and where we have supposed that the control switches $2 n+1$ times. We now define the following homotopy functions:

$$
\begin{aligned}
& H_{0}\left(y_{n+1}, \ldots, y_{1}, \lambda, \Delta_{n}, \ldots, \Delta_{1}\right) \\
& \quad=F_{0}\left(y_{n+1}, y_{n}, \ldots, y_{1}\right)+\lambda(1-\lambda) y_{n+1} \sum_{i=1}^{n} y_{i} \Delta_{i} \\
& H_{j}\left(y_{n+1}, \ldots, y_{1}, \lambda, \Delta_{n}, \ldots, \Delta_{1}\right)=\lambda F_{j}\left(y_{n+1}, y_{n}, \ldots, y_{1}\right) \\
& \quad+(1-\lambda)\left[2(-1)^{n-j+1} \cos \left(r_{j} y^{n-j+1}\right)+(-1)^{n+1}\right]
\end{aligned}
$$

where $j=1,2, \ldots, n$ and where $\lambda$ is the homotopy parameter, $0 \leq \lambda \leq 1$. Notice that $H_{j}\left(y_{n+1}, \ldots, y_{1}, 1, \Delta_{n}, \ldots, \Delta_{1}\right)=$ 
Table 4 Predicted and actual attitude errors comparison for $t \geq t_{f}^{\star} / 2$

\begin{tabular}{lllll}
\hline \hline The number of modes suppressed at $t_{f}^{*} / 2, n:$ & 0 & 1 & 2 & 3 \\
\hline Maximum pointing error (exact), deg: & 16.9760 & 2.1523 & 0.2625 & 0.0037 \\
Predicted upper bound using Eq. (50), deg: & 81.4671 & 9.7163 & 0.4052 & 0.0463 \\
\hline \hline
\end{tabular}

$0=F_{j}\left(y_{n+1}, y_{n}, y_{n-1}, \ldots, y_{2}, y_{1}\right)$ where $j=0,1,2, \ldots, n$. Inclusion of the additional parameters $\Delta_{1}, \Delta_{2}, \ldots, \Delta_{n}$, in Eq. (54) helps prevent ill-conditioning of Eqs. (54) and (55) as $y_{k} \rightarrow y_{k-1}$ or $y_{1} \rightarrow 0$. For example, if $y_{k} \rightarrow y_{k-1}$ then $\Delta_{k} \neq \Delta_{k-1} \neq 0$ and all other $\Delta_{i}$ are set to zero; if $y_{1} \rightarrow 0$ then $\Delta_{1} \neq 0$ and all other $\Delta_{i}$ are set to zero. The starting points of these homotopy maps, i.e., for $\lambda=0$, are as follows:

$$
y_{n-j+1}=\left(1 / r_{j}\right) \cos ^{-1}\left[(-1)^{j-1} / 2\right]
$$

where $j=1,2, \ldots, n$, and

$$
y_{n+1}=\left[P / \alpha-2 \sum_{i=1}^{n}(-1)^{i} y_{n-i+1}^{2}\right]^{0.5}
$$

For specified values of parameters $P, r_{2}, r_{3}, \ldots, r_{n}$ the homotopy path, starting at $\lambda=0$, is followed by numerically integrating the basic differential equations ${ }^{45}$ arising from Eqs. (54) and (55) until $\lambda \cong 1$, where $y_{j}$ and $j=1,2, \ldots, n+1$, are the solutions of Eqs. (52) and (53). These switching times are now substituted in Eq. (31) to construct a candidate costate vector. If this costate vector satisfies Eq. (32), then the solutions $y_{j}$ and $j=1,2, \ldots, n+1$ are optimal.

\section{Example Slewing Maneuvers}

Consider a flexible spacecraft with two uniform flexible appendages, whose material and maneuver specifications are given in Table 1. One torque actuator located on the rigid central body is used to control the slewing. The rigid body mode and the first 10 vibrational modes are retained in the evaluation model. The normalized mode shapes for a fixed-free cantilever beam are used as the assumed mode shapes. The results of the modal analysis appear in Table 2 . Four situations are now considered in which the first, the first two, the first three, and the first four vibrational modes are used, in addition to the rigid body mode, to define the control model; and hence are actively suppressed at the final time. The solutions of Eqs. (50) and (51) for a 90 deg rest-to-rest slewing maneuver corresponding to a parameter value $P=28.87059$ appear in Table 3 . These optimal switching and final times apply to a $90 \mathrm{deg}$ rest-to-rest maneuver centered about time $t=0$. It takes $9.338607 \mathrm{~s}$ to slew an equivalent rigid body through $90 \mathrm{deg}$. The maneuver times for maneuver starting at $t=0$ for cases where the first, the first two, the first three, and the first four modes are actively suppressed at the final time are found to be $9.45013,9.457802,9.458439$ and $9.458447 \mathrm{~s}$, respectively. All of these maneuvers are found to have begun with $u(t)=+U_{0}$ (i.e., $\alpha=+1$ ). The difference in maneuver times decreases as we increase the number of modes suppressed at the final time. Moreover since $\omega_{1}^{2} \theta_{f} / U_{0}=28.8706 \gg 1$, we observe that the time to slew an equivalent rigid body is not significantly different from the slewing times of any of the four cases.

The rigid hub would continue to oscillate after the maneuver has been completed due to the energy spillover to the residual modes. This results in a pointing error of the rigid central body. A bound on this pointing error can be determined. Using Eqs. (49-51) we are able to determine the number of modes that need to be actively controlled at the final time so that we are guaranteed a prespecified pointing accuracy after the maneuver has been completed [Eq. (50) applies here]. The exact maximum attitude error and the upper bound on the attitude error obtained using Eq. (50) are compared in Table 4. In this case

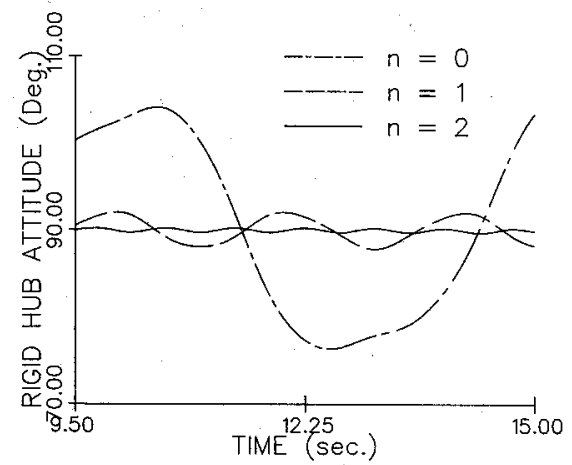

Fig. 7 The post-maneuver rigid central body attitude time history $(n=0,1,2)$.

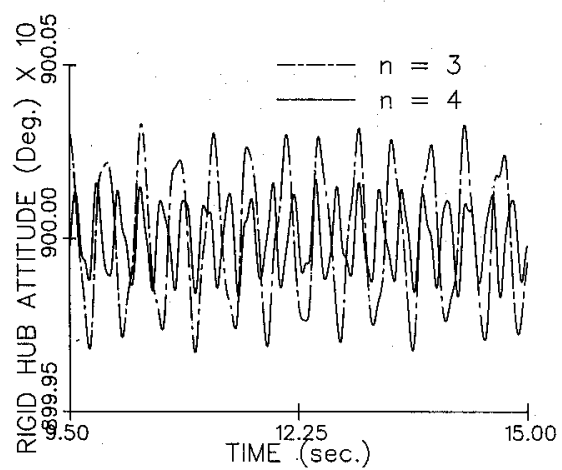

Fig. 8 The post-maneuver rigid central body attitude time history $(n=3,4)$.

[as per Eq. (50)], we need to control at least six modes (predicted accuracy of $0.0011 \mathrm{deg}$ ), so that we are guaranteed a pointing accuracy of better than $0.0019 \mathrm{deg}$. However, an accuracy of this magnitude is realized, in actuality, if we suppress only the first four modes at the final time. There appears to be a sizeable difference between the actual attitude errors and the attitude error predicted using Eq. (50). The difference, however, becomes smaller and smaller as more modes are actively suppressed at the final time. The agreement between the two quantities compared above is also dependent, somewhat, on the required pointing accuracy. For example, Eq. (50) predicts that we need to control two modes actively to be guaranteed an attitude accuracy better than $0.41 \mathrm{deg}$. In actuality, we must also control at least the first two modes to have a pointing error of less than $0.41 \mathrm{deg}$. The optimal control, energy, and the rigid hub attitude $(\theta)$ time histories during and after the maneuver for the five cases $(n=0,1,2,3,4)$ are presented in Figs. 2-6. Post maneuver time histories of the rigid central body attitude $(\theta)$ are magnified in Figs. 7 and 8 (cases shown correspond to $n=0,1,2$ and $n=3,4$, respectively). The residual energies for the cases examined are found to be $20.673,5.199,2.763,0.011$, and $0.014 \mathbf{J}$ (for $n=0,1,2,3$, and 4, respectively). It decreases initially as more modes are brought to rest at the end of the maneuver, but then it increases slightly as we suppress an additional mode at the final time. In contrast, the maximum pointing error of the central body decreases throughout, implying perhaps that it is the maximum pointing error and not the residual energy that is the proper measure of the maneuver accuracy. 


\section{Conclusions}

The time-optimal, single-axis, rest-to-rest rotational maneuver of an elastic spacecraft has been considered. The main results of the present work are as follows:

1) The optimal control history has an important time symmetry property.

2) The optimal switching times, the final time, and the costates at midmaneuver satisfy a system of nonlinear algebraic equations that can be solved using homotopy methods.

3) When only one control input is used, the optimal control history is independent of actuator locations (as described earlier).

4) Upper bounds on the attitude error of the central rigid body, arising from control spillover, are obtained.

The numerical optimization problem has been reduced to a system of nonlinear algebraic equations in the optimal switching times and the optimal final time. A homotopy method has been proposed to obtain solutions to these equations. Upper bounds on the pointing errors of the central body have been presented. These provide a rough estimate of the number of vibrational modes that need to be actively suppressed at the final time so that the pointing accuracy is better than a prespecified value. It is found that, if the maneuver parameter is sufficiently large, the slewing time for an equivalent rigid body provides a good estimate of the time required to slew the elastic spacecraft.

It is noticed that it is possible to achieve very fine pointing of an elastic spacecraft by controlling only a few of the elastic modes. In the example maneuver presented, it is seen that for a large angle rest-to-rest slewing maneuver, fine terminal pointing accuracy is achievable if the first four vibrational modes are actively suppressed.

There are, however, some limitations of the present work. The analysis here is restricted to linear, elastic, undamped, nongyroscopic systems. However, some natural damping is always present. The assumption regarding small angular velocities might fail to hold for minimum time rotational maneuvers unless the control limits are sufficiently small. Both of these assumptions, however, greatly simplify the analysis. The presence of damping destroys the symmetry property of optimal controls, which is one of the main results of this paper.

There are obvious implications of the results presented here. For example, there is a suggested separation of the time-optimal control problem and the structural optimization problem when only one control input is present. The time optimal control problem can be solved independently of the structural optimization problem of locating the actuators to optimize some structural excitation criteria (without affecting the optimal control history).

\section{Appendix}

Theorem 1: For the optimal control problem defined by Eqs. $(18-22)$, the optimal control $\boldsymbol{u}(t)^{*}$ is an odd function of time i.e., $\boldsymbol{u}(t)^{*}=-\boldsymbol{u}(-t)^{*},-t_{f}^{*} / 2 \leq t \leq t_{f}^{*} / 2$, where $t_{f}^{*}$ is the optimal maneuver time.

Proof: Normality of the pair $(\mathbf{A}, \mathbf{B})$ guarantees that an optimal control exists ${ }^{41,42}$ and is unique. The remainder of the proof is to demonstrate that the unique optimal control $u(t)^{*}$ satisfies the following symmetry property:

$$
\boldsymbol{u}(t)^{*}=-u(-t)^{*}
$$

where $-t_{f}^{*} / 2 \leq t \leq t_{f}^{*} / 2$.

Let $t_{f}^{*}, \boldsymbol{x}(t)^{*}, \boldsymbol{u}(t)^{*}$, and $t \in\left[-t_{f}^{*} / 2, t_{f}^{*} / 2\right]$ denote the optimal time, optimal state, and the optimal control. There is a vector function $p(t)=\left(p^{0}(t), q^{0}(t), p^{1}(t), q^{1}(t), \ldots, p^{n}(t), q^{n}(t)\right), \quad$ where $t \in\left[-t_{f}^{*} / 2, t_{f}^{*} / 2\right]$ such that:

$$
\dot{\boldsymbol{x}}(t)^{*}=\mathbf{A} \boldsymbol{x}(t)^{*}+\mathbf{B} \boldsymbol{u}(t)^{*}
$$

where $t \in\left[-t_{f}^{*} / 2, t_{f}^{*} / 2\right]$

$$
\dot{p}(t)=-\mathbf{A}^{\prime} \boldsymbol{p}(t)
$$

where $t \in\left[-t_{f}^{*} / 2, t_{f}^{*} / 2\right]$

$$
u_{j}(t)^{*}=-U_{j} \operatorname{sgn}\left[\sum_{i=0}^{n} \beta_{j}^{i} q^{i}(t)\right]
$$

where $j=0,1,2,3, \ldots, m$ and $t \in\left[-t_{f}^{*} / 2, t_{f}^{*} / 2\right]$

$$
\begin{gathered}
x\left(-t_{f}^{*} / 2\right)^{*}=\left(-\theta_{f} / 2,0,0,0, \ldots, 0\right)^{\prime} \\
x\left(t_{f}^{*} / 2\right)^{*}=\left(\theta_{f} / 2,0,0,0, \ldots, 0\right)^{\prime} \\
1+p(t)^{\prime}\left[\mathbf{A x}(t)^{*}+\mathbf{B} u(t)^{*}\right] \equiv 0
\end{gathered}
$$

where $t \in\left(-t_{j}^{*}\left|2, t_{f}^{*}\right| 2\right)$.

Existence and uniqueness theorems $\mathrm{s}^{41,42}$ guarantee that Eqs. (A2-A7) have the unique solution $t_{f}^{*}, x(t)^{*}, u(t)^{*}, p(t)$, and $t \in\left[-t_{f}^{*} / 2, t_{f}^{*} / 2\right]$.

Now consider the related time optimal control problem.

Minimize:

$$
\mathbb{J}=\int_{-t_{f / 2}}^{0} \mathrm{~d} t
$$

subject to:

$$
\ddot{x}(t)=\mathbf{A x}(t)+\dot{\mathrm{B} u}(t)
$$

where $t \in\left[-t_{f} / 2,0\right]$

$$
\left|u_{j}(t)\right| \leq U_{j}
$$

where $\mathrm{j}=0,1,2,3, \ldots, m$ and $t \in\left[-t_{f} / 2,0\right]$

$$
\begin{gathered}
x\left(-t_{f} / 2\right)=\left(-\theta_{f} / 2,0,0,0, \ldots, 0\right)^{\prime} \\
x(0) \in \mathscr{W}=\left\{\left(0, x_{2}^{L}, 0, x_{4}^{1 L}, 0, x_{4}^{2 L}, \ldots, 0, x_{4}^{n L}\right)^{\prime} \mid x_{2}^{L}, x_{4}^{i L} \in \mathbb{R}\right. \\
i=1,2, \ldots, n\}
\end{gathered}
$$

As in the original problem, it can be shown that this problem has a unique optimal solution denoted by $t_{L}^{*}, \boldsymbol{x}_{L}(t)^{*}, \boldsymbol{u}_{L}(t)^{*}$, and $t \in\left[-t_{L}^{*} / 2,0\right]$. There is a vector function $p_{L}(t)=$ $\left[p_{0}^{L}(t), q_{0}^{L}(t), p_{1}^{L}(t), q_{1}^{L}(t), \ldots, p_{n}^{L}(t), q_{n}^{L}(t)^{\prime}\right]$, and $t \in\left[-t_{L}^{*} / 2,0\right]$ such that:

$$
\dot{\boldsymbol{x}}_{L}(t)^{*}=\mathbf{A} \boldsymbol{x}_{L}(t)^{*}+\mathbf{B} u_{L}(t)^{*}
$$

where $t \in\left(-t_{L}^{*} / 2, t_{L}^{*} / 2\right)$

$$
\dot{p}_{L}(t)=-A^{\prime} p_{L}(t)
$$

where $t \in\left[-t_{L}^{*} / 2,0\right]$

$$
u_{j L}(t)^{*}=-U_{j} \operatorname{sgn}\left[\sum_{i=0}^{n} \beta_{j}^{i} q_{L}^{i}(t)\right]
$$

where $j=0,1,2,3, \ldots, m$ and $t \in\left[-t_{L}^{*} / 2,0\right]$

$$
\boldsymbol{x}_{L}\left(-t_{L}^{*} / 2\right)^{*}=\left(-\theta_{f} / 2,0,0,0, \ldots, 0\right)^{\prime}
$$

$$
x_{L}(0) * \in \mathscr{W}
$$

$$
p_{L}(0) \in\left[\left(p_{0}^{L}, 0, p_{1}^{L}, 0, p_{2}^{L}, 0, \ldots, p_{n}^{L}, 0\right)^{\prime} \mid p_{i}^{L} \in \mathbb{R}, i=0,1, \ldots, n\right]
$$

$$
1+p_{L}(t)^{\prime}\left[\mathrm{A} x_{L}(t)^{*}+\mathbf{B} u_{L}(t)^{*}\right]=0,
$$

where $t \in\left(-t_{L}^{*} / 2,0\right)$ 
Now define:

$$
u(t)=u_{L}(t)^{*}
$$

where $t \in\left[-t_{L}^{*} / 2,0\right]$ and

$$
u(t)=-u_{L}(-t)^{*}
$$

where $t \in\left[0, t_{L}^{*} / 2\right]$, and where $x(t), p(t)=\left[p^{0}(t), q^{0}(t), p^{1}(t)\right.$, $\left.q^{1}(t), \ldots, p^{n}(t), q^{n}(t)\right]^{\prime}$, and $t \in\left[-t_{L}^{*} / 2, t_{L}^{*} / 2\right]$ by

$\dot{\boldsymbol{x}}(t)=\mathbf{A} \boldsymbol{x}(t)+\mathbf{B} \boldsymbol{u}(t), \quad \boldsymbol{x}\left(-t_{L}^{*} / 2\right)=\left(-\dot{\theta}_{f} / 2,0,0,0, \ldots, 0\right)^{\prime}$

$$
\dot{p}(t)=-\mathbf{A}^{\prime} p(t), \quad p(0)=p_{L}(0)
$$

It is straightforward to show that:

$$
\begin{gathered}
x\left(t_{L}^{*} / 2\right)=\left(\theta_{f} / 2,0,0,0, \ldots, 0\right)^{\prime} \\
u_{j}(t)=-U_{j} \operatorname{sgn}\left[\sum_{i=0}^{n} \beta_{j}^{i} q^{i}(t)\right]
\end{gathered}
$$

where $j=0,1,2,3, \ldots, m$ and $t \in\left[-t_{L}^{*} / 2, t_{L}^{*} 2\right]$

$$
1+\boldsymbol{p}(t)^{\prime}(\mathbf{A x}(t)+\mathbf{B} \boldsymbol{u}(t)) \equiv 0
$$

where $t \in\left(-t_{L}^{*} / 2, t_{L}^{*} / 2\right)$,

By construction, therefore, $t_{L}^{*}, x(t), u(t), p(t)$, and $t \in\left[-t_{L}^{*} / 2\right.$, $\left.t_{L}^{*} / 2\right]$ satisfy Eqs. (A2-A7). The uniqueness of the solutions of Eqs. (A2-A7) implies that

$$
\begin{gathered}
t_{f}^{*}=t_{L}^{*} \\
x(t)^{*}=\dot{x}(t)
\end{gathered}
$$

where $t \in\left[-t_{f}^{*} / 2, t_{f}^{*} / 2\right]$

$$
p(t)^{*}=p(t)
$$

where $t \in\left[-t_{f}^{*} / 2, t_{f}^{*} / 2\right]$ and

$$
u(t)^{*}=u(t)
$$

where $t \in\left[-t_{f}^{*} / 2, t_{f}^{*} / 2\right]$

Equations (A19) and (A20) then imply that

$$
\boldsymbol{u}(t)^{*}=-\boldsymbol{u}(-t)^{*}
$$

where $t \in\left[-t_{f}^{*} / 2, t_{f}^{*} / 2\right]$

Theorem 2: Suppose that for some integers $k^{j}$, and $0 \leq j \leq m$ Eq. (29-31) admit a solution $\left(t_{r}^{j}, 1 \leq r \leq k^{j} ; 0 \leq j \leq m ; t_{f} / 2 ; p_{0}^{i}\right.$, $0 \leq i \leq n$ ) satisfying inequalities in Eqs. (27) and (32). Then:

1) The $j$ th component of the optimal control solving problem (18-22) switches exactly $2 k^{j}+1$ times at instants $-t_{k j}^{j},-t_{k j-1}^{j}, \ldots,-t_{1}^{j}, 0, t_{1}^{j}, \ldots, t_{k j-1}^{j}, t_{k j}^{j}$.

2) The optimal maneuver time of the optimal control solving problem (18-22) is exactly $t_{f}$.

Proof: Suppose that for some integers $k^{j}, 0 \leq j \leq m$, Eqs. (29) and (30) admit a solution $\left(t_{r}^{j}, 1 \leq r \leq k^{j ;} 0 \leq j \leq m ; t_{f} / 2\right.$; $p_{0}^{i}, 0 \leq i \leq n$ ) satisfying inequalities in Eq. (27). Using this solution in Eq. (31) we obtain the costates at midmaneuver and hence obtain $p(t) \in \mathbb{R}^{2 n+2}$, satisfying Eqs. (23) and (26). Using this $p(t)$, we define $u(t) \in \mathbb{R}^{m+1}$ by $u_{j}(t)=-U_{j} \operatorname{sgn}\left(p(t)^{\prime} b_{j}\right)$, $0 \leq j \leq m,-t_{f} / 2 \leq t \leq t_{f} / 2$. It is easily verified that the functions $\boldsymbol{p}(t)^{\prime} \boldsymbol{b}_{j}$ vanish exactly at instants $-t_{k^{j}}^{j},-t_{k^{j}-1}^{j}, \ldots,-t_{1}^{j}$, $0, t_{1}^{j}, \ldots, t_{k^{j}-1}^{j^{j}}, t_{k^{j}}^{j} ; 0 \leq j \leq m$. Moreover, between $-t_{f} / 2$ and $t_{f} / 2$, these functions have no other zeros because of Eq. (32). Therefore, the preceding $u(t)$ is a piecewise constant such that $u_{j}(t)$ switches at instants $\left( \pm t_{r}^{j}, 1 \leq r \leq k^{j} ; 0\right)$. Integrating Eq. (18) with initial conditions [first of Eq. 22] yields:

$$
\boldsymbol{x}\left(t_{f} / 2\right)=\left[\begin{array}{c}
\sum_{j=0}^{m} U_{j} \alpha_{j}\left\{\left(t_{f} / 2\right)^{2}-2\left(t_{k^{j}}\right)^{2}+\cdots+2(-1)^{k^{j}}\left(t_{1}^{j}\right)^{2}\right\}-\theta_{f} / 2 \\
-\left(2 / \omega_{1}\right) \sum_{j=0}^{m} \alpha_{j} \beta_{j}^{1} U_{j}\left\{\cos \left(\omega_{1} t_{f} / 2\right)-\cdots+2(-1)^{k^{j}} \cos \left(\omega_{1} t_{1}^{j}\right)+(-1)^{k^{j}+1}\right\} \cos \left(\omega_{1} t_{f} / 2\right) \\
\left(2 / \omega_{1}\right) \sum_{j=0}^{m} \alpha_{j} \beta_{j}^{1} U_{j}\left\{\cos \left(\omega_{1} t_{f} / 2\right)-\cdots+2(-1)^{k^{j}} \cos \left(\omega_{1} t_{1}^{j}\right)+(-1)^{k^{j}+1}\right\} \sin \left(\omega_{1} t_{f} / 2\right) \\
-\left(2 / \omega_{2}\right) \sum_{j=0}^{m} \alpha_{j} \beta_{j}^{2} U_{j}\left\{\cos \left(\omega_{2} t_{f} / 2\right)-\cdots+2(-1)^{k^{j}} \cos \left(\omega_{2} t_{1}^{j}\right)+(-1)^{k^{j}+1}\right\} \cos \left(\omega_{2} t_{f} / 2\right) \\
\vdots \\
-\left(2 / \omega_{2}\right) \sum_{j=0}^{m} \alpha_{j} \beta_{j}^{2} U_{j}\left\{\cos \left(\omega_{2} t_{f} / 2\right)-\cdots+2(-1)^{k^{j}} \cos \left(\omega_{2} t_{1}^{j}\right)+(-1)^{k^{j}+1}\right\} \sin \left(\omega_{2} t_{f} / 2\right) \\
\left(2 / \omega_{n}\right) \sum_{j=0}^{m} \alpha_{j} \alpha_{j} \beta_{j}^{n} U_{j}\left\{\cos \left(\omega_{n} t_{f} / 2\right)-\cdots+2(-1)^{k^{j}} \cos \left(\omega_{n} t_{1}^{j}\right)+(-1)^{k^{j}+1}\right\} \sin \left(\omega_{n} t_{f} / 2\right)
\end{array}\right]
$$

But because $t_{r}^{j}, 1 \leq r \leq k^{j}, 0 \leq j \leq m, t_{f} / 2$ satisfy Eqs. (29) and (30), Eq. (A24) yields $\left.x\left(t_{f} / 2\right)=\theta_{f} / 2,0,0,0, \ldots, 0,0\right)^{\prime}$, the desired final state. As a consequence, the $p(t)$ and $u(t)$ just constructed satisfy all the necessary and sufficient conditions for optimality in Eqs. (18-26) and are therefore the optimal costate and control, respectively, i.e.

$$
t_{f}^{*}=t_{f}, \quad t_{r}^{j *}=t_{r}^{j}, \quad 1 \leq r \leq k^{j} ; \quad 0 \leq j \leq m
$$


Corollary 2: Suppose that integers $k^{j}, 0 \leq j \leq m$ satisfy $\sum_{j=0}^{m} j^{k}=n$. Also assume that Eqs. (29) and (30) admit a solution $\left(t_{r}^{j}, 1 \leq r \leq k^{j} ; 0 \leq j \leq m ; t_{f} / 2\right)$ which is regular ${ }^{43}$ and satisfies Eqs. (27), (31), and (32). Then the claims of Theorem 2 are true.

Proof: Suppose Eqs. (29) and (30) admit a regular solution as assumed. Consider the vector equation (31) for $\left(p_{0}^{0}, p_{0}^{1}, \ldots, p_{0}^{n}\right)^{\prime}$. Rewriting Eqs. (29) and (30) as

$$
\sum_{j=0}^{m} \alpha_{j} U_{j}\left[\left(t_{f} / 2\right)^{2}-2\left(t_{k^{j}}^{j}\right)^{2}+2\left(t_{k^{j}-1}^{j}\right)^{2}-\cdots+2(-1)^{k^{j}}\left(t_{1}^{j}\right)^{2}\right]=\theta_{f}
$$

$$
\begin{array}{r}
\left(-2 / \omega_{i}\right) \sum_{j=0}^{m} \alpha_{j} \beta_{j}^{i} U_{j}\left[\cos \left(\omega_{i} t_{f} / 2\right)-2 \cos \left(\omega_{i} t_{k}^{j}\right)\right. \\
\left.+\cdots+2(-1)^{k^{j}} \cos \left(\omega_{i} t_{1}^{j}\right)+(-1)^{k^{j}+1}\right]=0
\end{array}
$$

where $i=1,2, \ldots, n$. It is seen that the coefficient matrix in Eq. (31) is exactly the transpose of the jacobian of Eqs. (A26) and (A27). Regularity of the solution $t_{r}^{j}, 1 \leq r \leq k^{j}, 0 \leq j \leq m$, and $t_{f} / 2$ of Eqs. (29) and (30) implies the existence and uniqueness of the solution of Eq. (31). Using this solution, a costate vector $p(t)$ satisfying Eq. (23) can be constructed. Satisfaction of Eq. (32) by the costate vector thus obtained implies that all of the necessary and sufficient conditions are met. The costate thus constructed is therefore the optimal costate and the optimal control is characterized by the regular solution of Eqs. (29) and (30).

\section{References}

${ }^{1}$ Meyer, G., "Design and Global Analysis of Spacecraft Attitude Control Systems," NASA TR R-361, March 1971.

${ }^{2}$ Meyer, G., "On the Use of Euler's Theorem on Rotations for the Synthesis of Attitude Control Systems," NASA TN D-3643, July 1966.

${ }^{3}$ Meyer, G., "Response Envelope - A Global Description of 3-Axis Large Angle Spacecraft Attitude Control Systems," NASA TN D4896, June 1968.

${ }^{4}$ Kranton, J., "Minimum Time Attitude Maneuvers with Control Movement Gyros," AIAA Journal, Vol. 8, Aug. 1970, pp. 1523-1525.

${ }^{5}$ Dixon, M. V., Edelbaum, T. N., Potter, J. E., and Vander Velde, W. E., "Fuel Optimal Reorientations of Axisymmetrix Spacecraft," Journal of Spacecraft and Rockets, Vol. 7, Nov. 1970, pp. 1345-1351.

${ }^{6}$ Bergman, E. E., Croopnick, S. R., Turkovich, J. J., and Work, C. C., "An Advanced Spacecraft Autopilot Concept," Journal of Guidance and Control, Vol. 2, May-June 1979, pp. 161-168.

${ }^{7}$ D'Amario, L. A. and Stubbs, G. S., "A New Single Rotation Axis Autopilot for Rapid Spacecraft Attitude Maneuvers," Journal of Guidance and Control, Vol. 2, July-Aug. 1979, pp. 339-346.

${ }^{8} \mathrm{Chen}, \mathrm{J}$. and Kane, T. R. "Slewing Maneuvers of Gyrostat Spacecraft," Journal of Astronautical Sciences, Vol. 28, July-Sept. 1980, pp. 267-281.

${ }^{9}$ Carrington, C. K. and Junkins, J. L., "Nonlinear Feedback Control of Spacecraft Slewing Maneuvers," Advances in Astronautial Sciences, AAS Paper 83-002, 1983.

${ }^{10}$ Vadali, S. R. and Junkins, J. L., "Spacecraft Large Angle Rotational Maneuvers with Optimal Momentum Transfer," Journal of Astronautial Sciences, Vol. 31, April-June 1983, pp. 217-235.

${ }^{1}$ Dywer, T. A. W., III "Exact Nonlinear Control of Large Angle Rotational Maneuvers," IEEE Transactions of Automatic Control, Vol. AC-29, Sept. 1984, p. 769.

${ }^{12}$ Dywer, T. A. W., III and Batten, A. J., "Exact Spacecraft Detumbling and Reorientation Maneuvers with Gimbaled Thrusters and Reaction Wheels," Journal of Astronautial Sciences, Vol. 33, April-June 1985, pp. 217-232.

${ }^{13}$ Dywer, T. A. W., III "Exact Nonlinear Control of Spacecraft Slewing Maneuvers with Internal Momentum Transfer," Journal of Guidance and Control, Vol. 9, March-April 1986, pp. 240-247.

${ }^{14}$ Dywer, T. A. W., III and Sena, R. P., "Control of Spacecraft Slewing Maneuvers," Proceedings of the IEEE Conference on Decision \& Control, IEEE, New York, 1982, Vol. 3, pp. 1141-1144.

${ }^{15}$ Skaar, S. B. and Kraige, L. G., "Large Angle Spacecraft Attitude Maneuvers using an Optimal Reaction Wheel Power Criterion," Journal of Astronautial Sciences, Vol. 32, Jan.-March 1984, pp. 47-61

${ }^{16}$ Vadali, S. R. and Junkins, J. L., "Optimal Open Loop and Stable
Feedback Control of Rigid Spacecraft Attitude Maneuvers," Journal of Astronautial Sciences, Vol. 32, April-June 1984, pp. 105-122.

${ }^{17}$ Wie, B. and Barba, P. M., "Quaternion Feedback for Spacecraft Large Angle Maneuvers," Journal of Guidance, Control and Dynamics, Vol. 8, May-June 1985, pp. 360-365.

${ }^{18}$ Vadali, S. R., "Variable Structure Control of Spacecraft Large Angle Maneuvers," Journal of Guidance, Control, and Dynamics, Vol. 9, March-April 1986, pp. 235-239.

${ }^{19}$ Markley, F. L., "Large Angle Maneuver Strategies for Flexible Spacecraft," Advances in Astronautial Sciences, AAS Paper 79-156, 1979.

${ }^{20}$ Farrenkopf, R. L., "Optimal Open Loop Maneuver Profiles for Flexible Spacecraft," Journal of Guidance and Control, Vol. 3, Nov.Dec. 1979, pp. 491-498.

${ }^{21}$ Swigert, C. J., "Shaped Torque Techniques," Journal of Guidance and Control, Vol. 3, Sept.-Oct. 1980, pp. 460-467.

${ }^{22}$ Turner, J. D. and Junkins, J. L., "Optimal Large Angle Single Axis Rotational Maneuvers of Flexible Spacecraft," Journal of Guidance and Control, Vol. 3, Nov.-Dec. 1980, pp. 578-585.

${ }^{23}$ Turner, J. D., Chun, H. M., and Junkins, J. L., "Optimal Large Angle Maneuvers with Active Vibration Suppression," NASA CP2258, Feb. 1983, pp. 177-215.

${ }^{24}$ Turner, J. D., Chun, H. M., and Juang, J., "Closed Form Solutions for a Class of Optimal Quadratic Regulator Problems with Terminal Constraints," Transactions of the American Society of Mechanical Engineers, Vol. 108, March 1986, pp. 44-48.

${ }^{25}$ Juang, J,, Turner, J. D., and Chun, H. M., "Large Angle Maneuvers of Flexible Spacecraft Using a Closed Form Solution for the Terminal Tracking Problem," AAS Paper 83-375, 1983.

${ }^{26}$ Turner, J. D. and Chun, H. M., "Optimal Distributed Control of a Flexible Spacecraft During a Large Angle Maneuver," Journal of Guidance, Control, and Dynamics, Vol. 7, May-June 1984, pp. 257-264.

${ }^{27}$ Chun, H. M., Turner, J. D., and Juang, J., "Disturbance Accommodating Tracking Maneuvers of Flexible Spacecraft," Journal of Astronautial Sciences, Vol. 33, April-June 1985, pp. 197-216.

${ }^{28}$ Skaar, S. B. and Tucker, D., "Optimal Control of Flexible Systems Using a Convolution Integral Description of Motion," Transactions of the IEEE Conference on Decision and Control, 1983, pp. 825-829.

${ }^{29}$ Skaar, S. B. and Tucker, D., "Point Control of a One-Link Flexible Manipulator," Journal of Applied Mechanics, Vol. 53, March 1986, pp. 23-27.

${ }^{30}$ Lisowski, R. J. and Hale, A. L., "Optimal Design for Single Axis Rotational Maneuvers of a Flexible Structure," Journal of Astronautial Sciences, Vol. 32, April-June 1985, pp. 179-196.

${ }^{31}$ Hale, A. L. and Lisowski, R. J., "Characteristic Elastic Systems of Time Limited Optimal Maneuvers," Journal of Guidance, Control, and Dynamics, Vol. 8, Sept.-Oct. 1985, pp. 628-636.

${ }^{32}$ Breakwell, J. A., "Optimal Feedback Slewing of Flexible Spacecraft," Journal of Guidance and Control, Vol. 4, Sept.-Oct. 1981, pp. 472-479.

${ }^{33}$ Dwyer T. A. W. III and Lee G. K. F., "Exact Nonlinear Command Generation and Tracking for Robot Manipulators and Spacecraft Slewing Maneuvers," Transactions of the IEEE Conference on Decision and Control, 1983, pp. 710-715.

${ }^{34}$ Juang, J., Horta, L. G., and Robertshaw, H. H., "A Slewing Control Experiment for Flexible Structure," Journal of Guidance, Control, and Dynamics, Vol. 9, Sept.-Oct. 1986, pp. 599-607.

${ }^{35}$ Vander Velde, W. E. and He. J., "Design of Space Structure Control System Using On-Off Thrusters,"' Journal of Guidance and Control, Vol. 6, Jan.-Feb. 1983, pp. 759-775.

${ }^{36}$ Floyd, M. A., Brown, M. E., Turner, J. D., and Vander Velde, W. E., "Implementation of a Minimum Time and Fuel On-Off Thruster Control System for Flexible Spacecraft," AAS Paper 83-376.

${ }^{37}$ Skaar, S. B., Tang, L., and Yalda-Mooshabad, Y., "On-Off Attitude Control of Flexible Satellites," Journal of Guidance, Control, and Dynamics, Vol. 9, July-Aug. 1986, pp. 507-510.

${ }^{38}$ Thompson, R. C., Junkins, J. L., and Vadali, S. R., "Near Minimum Time Open Loop Control of Dynamics Systems," AIAA Paper 87-0598.

${ }^{39}$ Monaco, S. and Stronelli, S., "A Nonlinear Attitude Control Law for Satellite with Flexible Appendages," Proceedings of the 24th Conference on Decision and Control, Dec. 1985, pp. 1654-1659.

${ }^{40}$ Greenwood, D. T., Classical Dynamics, Prentice-Hall, Englewood Cliffs, NJ, 1977.

${ }^{41}$ Lee, E. B. and Marcus, L., Foundations of Optimal Control Theory, Wiley, New York, 1967.

${ }^{42}$ Hermes, H. and LaSalle, J., Functional Analysis and Time Optimal Control, Academic, New York, 1969.

${ }^{43}$ Garcia, C. B. and Zangwill, W. I., Pathways to Solutions, Fixed Points and Equilibria, Prentice-Hall, Englewood Cliffs, NJ, 1981. 